\title{
MONASTERI E ATTIVITÀ MINERARIA NELL'ITALIA ALTO MEDIEVALE. SUGGERIMENTI E PROBLEMI
}

\section{VASCO LA SALVIA}

UDC: $27-788(35)$

$622+669(35)$

Review

Manuscript received: 01. 11. 2016.

Revised manuscript accepted: 07. 02. 2017.

DOI: 10.1484/J.HAM.5.113727
V. La Salvia

Dipartimento di Scienze psicologiche, della salute e del territorio Università degli Studi "G. D’Annunzio"

Chieti - Pescara, Italy

vasco.lasalvia@unich.it | vlasalvia@unich.it

The paper attempts to investigate, through the study of iron production, particularly in central and northern Italy, the development of the historical forms of property in close relationship with the unraveling control of the elites on the countryside. The entire set of sources (both archaeological and written), for the period under examination, seems to indicate that production was now strongly orientedlfocused to meet the overriding interests of large landed property, a process to which the economic reorganization of the monasteries was certainly not alien but, on the contrary, it appears to have played a key role in the definition of new ways to control access to raw materials and to the territory in general.

Key words: early medieval economy, carolingian period, archaeometallurgy, mining industry, iron production, monasteries and real estate, agricultural tools.

Questo breve contributo riprende una serie di temi che, inizialmente vennero trattati ed ampiamente analizzati già nel secolo scorso attraverso i lavori di diversi colleghi, fra cui vale la pena menzionare Baruzzi, Fumagalli, Farinelli e Francovich, nel tentativo di tracciare le linee fondamentali dello sviluppo non solo delle attività agricole e minerarie ma, anche (o, probabilmente, soprattutto), quello delle forme storiche della proprietà in stretta relazione con il dipanarsi del controllo delle élites sulle campagne ${ }^{1}$.

Per il VII e l'VIII secolo dati archeologici e fonti scritte concorrono a presentare un quadro all'interno del quale $\mathrm{i}$ fabbri sembrano avere un ruolo rilevante nei rispettivi gruppi di appartenenza e nel corpo sociale longobardo. La situazione documentata dalle carte dell'epoca suggerisce, infatti, che nella società dell'Italia longobarda si fosse ormai da tempo stabilizzata una classe di artigiani specializzati in possesso di un bagaglio tecnico e operativo sufficientemente complesso. Ciò, peraltro, è confermato dalla qualità e quantità dei ritrovamenti e dei reperti rinvenuti nelle tombe di fabbri (quali quelle di Grupignano-Cividale, 224 Leno, 12 Centallo, 37 di Castel Trosino) e nelle botteghe e/o centri di produzione, lasciando supporre che tale processo di sedimentazione delle conoscenze tecnico-operative fosse iniziato assai prima dell'ingresso di questa popolazione nella Penisola. La fine di questo percorso è presentato dalle carte dell'VIII secolo, quando gli artigiani del metallo sono menzionati dalle fonti scritte come uomini liberi, vires honesti, e spesso si trovano impegnati in transazioni commerciali ed immobiliari ${ }^{2}$.

Nel corso del successivo periodo, durante il IX-X secolo, ovvero per la piena età carolingia, il panorama della produzione siderurgica sembra, invece, mutare 'scivolando' in modo sempre più significativo dall'iniziativa di maestranze

\footnotetext{
${ }^{1}$ Fra la sterminata bibliografia specifica in materia, si tengano presenti, V. FUMAGALLI, Storia agraria e luoghi comuni, in Studi storici, s. 3, 9, 1968, p. 949-965; ID., Note per una storia agraria altomedievale, in Studi storici, s. 3, 9, 1968, p. 359-378; ID., Terra e società nell'Italia Padana. I secoli IX eX, Torino, 1976; ID., Precarietà dell'economia contadina e affermazione della grande azienda fondiaria nell'Italia settentrionale dall'VIII all'XI secolo, in Rivista di storia dell'agricoltura, 15, 1976, p. 3-27; R. FARINELLI E R. FRANCOVICH, Paesaggi minerari della Toscana medievale. Castelli e metalli, in Castrum 5. Archéologie des espaces agraires méditerranéens au Moyen Âge, Roma-Madrid-Murcia, 1999, p. 467-488; R. FARINELLI, Gli statuti minerari cinquecenteschi di Massa Marittima, in Ricerche storiche, XL, 2010, p. 125-219; R. Francovich (ed.), Archeologia delle attività estrattive e metallurgiche, Firenze, 1993; R. FRANCOVICH, Per una storia sociale delle attività estrattive e metallurgiche: a proposito di alcune recenti ricerche archeologiche nella Toscana mineraria del Medioevo, in Actas de las I Jornadas sobre Minería y Tecnología en le Edad Media Peninsular, Léon, 1996, p. 19-35; M. BARUZZI, I reperti in ferro dello scavo di villa Clelia (Imola). Note sull'attrezzatura agricola nell'Altomedioevo, in R. Francovich (ed.), Archeologia e storia del Medioevo italiano, Roma, 1987, p. 151-170; M.E. CORTESE, L'acqua, il grano, il ferro. Opifici idraulici medievali nel bacino Farma-Merse, Firenze, 1997; V. LA SALVIA, Gap or Continuity? Mining in Early Medieval Italy, in G. Magnusson (ed.), The Importance of Ironmaking: Technical Innovation and Social Changes. Papers presented at the Norgberg Conference on May 8-13, 1995, Stockholm, 1995, p. 262-271.

${ }^{2}$ Sulle sepolture di fabbri di epoca longobarda vedi G.C. Menis (ed.), I Longobardi, Milano, 1990, p. 20-21, 32-33, 372; M. BROZZI, Strumenti di orafo longobardo, in Quaderni ticinesi/(NAC), 1, 1972, p. 167-74; V. LA SALVIA, Iron Making during the Migration Period. The Case of the Lombards, Oxford (BAR I.S 1715), 2007, p. 31; E. MICHELETTO e L. PEJRANI BARICCO, Archeologia funeraria ed insediativa in Piemonte tra Ve VII secolo, in L. Paroli (a cura di), L'Italia centro-settentrionale in età longobarda, Firenze, 1997, p. 315- 316, 334-35; P.M. DE MARCHI e A. BREDA, Il territorio bresciano in età longobarda e la necropoli di Leno, in C. Bertelli e G.P. Brogiolo (eds.), Il futuro dei Longobardi. L'Italia e la costruzione dell'Europa di Carlo Magno, Ginevra-Milano, 2000, p. 473, 479, 488-9o; L. PAROLI, Aspetti e problemi dell'archeologia della produzione in età longobarda, in M.S. Arena e L. Paroli (eds.), Le arti del fuoco in età longobarda, Roma, 1994, p. 14-15; L. PAROLI, La Necropoli di Castel Trosino: un laboratorio archeologico per lo studio dell'età longobarda, in L. Paroli (ed.), L'Italia centro-settentrionale in età longobarda, Firenze, 1997, p. 91; M. RICCI, Relazioni culturali e scambi commerciali nell'Italia centrale romano-longobarda alla luce della Crypta Balbi in Roma, in L. Paroli (ed.), L'Italia centro-settentrionale in età longobarda, Firenze, 1997, p. 253-56, 270; sullo sviluppo della 'figura' del fabbro nell'Europa alto medievale, vedi V. LA SALVIA e M. VALENTI, Tradizioni sepolcrali e luoghi di culto nel 'Barbaricum' fra identità etniche e sociali, in C. Ebanista e M. Rotili (eds.), Territorio, insediamenti e necropoli fra tarda Antichità e alto Medioevo, Napoli, 2016, p. 498-507.
} 
e singoli artigiani liberi, qualificati sempre come magistri, e, dunque, come uomini pienamente liberi, per quanto sempre sottoposti alla contrattazione diretta con la loro committenza, verso sistemi di organizzazione produttiva assai più rigidi e centralizzati che vedono molto frequentemente l'autorità ecclesiastico-monastica presentarsi come principale attore del controllo e della gestione delle risorse territoriali all'interno di un contesto sociale nel quale anche la diretta azione delle aristocrazie locali non pare essere stata ininfluente nel ridefinire gli assetti della gestione di patrimoni e risorse naturali. In particolar modo, le fonti scritte forniscono ripetuti esempi di questo fenomeno economico-sociale, consentendo di rintracciare le radici di questo processo di accentramento della ricchezza a partire dalla fine del secolo VIII quando sembra iniziare ad evidenziarsi il trasferimento di porzioni di patrimoni fondiari di origine pubblica nelle mani dell'aristocrazia e, soprattutto, dell'alta gerarchia ecclesiastica e monastica.

Un caso eclatante che interessa direttamente la produzione metallurgica è quello delle fondazioni monastiche lucchesi della fine del secolo VIII, zona in cui il contrasto fra aristocrazia locale e corona regia in questo periodo deve aver favorito certamente tali dinamiche: la volontà di includere il territorio che collega Monteverdi a Massa Marittima, una zona ricca in risorse minerarie e boschi, all'interno della iudiciaria lucense da parte dell'aristocrazia lucchese è attestata proprio dalla fondazione dei monasteri di S. Pietro di Monteverdi e S. Regolo e dimostra, da un lato, l'interesse delle élites urbane lucchesi nel controllo di quella porzione specifica di territorio e, dall'altro, come questo interesse fosse giustificato o giusrtificabile dalla possibile persistenza, per la medesima area, di attività minerarie e di trasformazione del metallo. Il primo cenobio, fondato nel 753, sembra nascere proprio con lo scopo di controllare importanti giacimenti di metalli preziosi presenti nei possessi di alcuni esponenti dell'aristocrazia lucchese e pisana, già detentori di uffici pubblici ma, all'epoca, in forte dissidio con l'autorità regia pavese. L'abbazia ebbe, infatti, sede in un'area marginale, il Cornino, situata in iudicaria Lucensi e al confine tra la diocesi di Populonia e quella volterrana.

Il sito prescelto per il monastero era prossimo ad una delle maggiori concentrazioni di "oro invisibile" della Toscana (l'area di Monterotondo) e collocato al centro di ricchi distretti minerari (il Massetano, l'alta Val di Cornia e il Campigliese), entro i quali deteneva un vasto patrimonio fondiario. Una connessione di questa istituzione religiosa con lo sfruttamento delle risorse minerarie appare ancor più probabile quando si consideri la contestuale fondazione del monastero femminile di S. Salvatore a Pitiliano sulla riva del fiume Versilia, vale a dire nello scalo marittimo naturale delle più importanti miniere apuane. Il secondo, invece, venne fondato proprio nel distretto minerario massetano ove si estendeva una vasta area fiscale, il waldus regis, in cui ancora nel 78o si trovava un maior selvani, al cui interno venne posizionato l'importante monastero. Rimanendo in Toscana, nel corso dell'età carolingia, censi in ferro sono percepiti in alcune curtis di questa regione e indicano la continuità, durante l'alto Medioevo, dello sfruttamento dei giacimenti locali e, probabilmente, di una produzione condotta da manentes. A tal proposito, vanno ricordati i quattro vomeri versati all'episcopato lucchese da Liutchari, un abitante di Custodia, sito localizzato lungo le propaggini settentrionali del monte pisano e i bomeri e le ferramenta, ottenuti tra IX eX secolo, dal monastero di S. Salvatore sul Monte Amiata. Quest'ultima situazione è assai interessante non solo in quanto nuovamente specifica l'ammontare del censo, qualificandolo come strumentazione agricola ma, soprattutto, in quanto è relativa al un livello che riguarda un mulino, un molendinum idraulico, che sorgeva nelle vicinanze dei giacimenti metalliferi. A livello indiziario, come fu notato a suo tempo da Farinelli e Francovich, potrebbe ipotizzarsi per questa zona una precoce stabilizzazione dell'utilizzo dell'energia idraulica anche per la produzione siderurgica, assai prima di quanto, dunque, tradizionalmente indicato dalle fonti scritte per i secoli XII-XIII e, sottolineando, una volta ancora, l'importanza della 'imprenditoria monastica' nello sviluppare nuove tecnologie o, quantomeno, nel disseminarne l'applicazione 3 .

Anche dall'Italia settentrionale provengono numerose indicazioni del diretto coinvolgimento dell'autorità monastica nella riorganizzazione della produzione metallurgica e della proprietà terriera. Alcune fra le maggiori fondazioni dell'epoca alto medievale, quali S. Giulia di Brescia, Bobbio, Nonantola, sono, infatti, citate dai documenti coevi quali attori principali di questo processo economico di accentramento della proprietà e ristrutturazione dei cicli produttivi. Il monastero di Santa Giulia, fondazione regia di epoca longobarda di fine secolo VIII, fra IX eX secolo riceveva dalle sue corti, collocate nelle valli del bresciano, ingenti quantitativi di ferro per anno, nella maggior parte sotto forma di strumenti agricoli. Non è, dunque, privo di importanza notare, in questa sede, che alcuni dei possedimenti del monastero insistono all'interno di un'area geografica che fin dall'epoca tardo antica si segnala, sia dal punto di vista archeologico che delle fonti scritte, come fortemente interessata dall'attività metallurgica; inoltre, nel periodo longobardo proprio presso Leno occorre menzionare la presenza della tomba di un fabbro (la 224) all'interno di una necropoli che ha restituito grandi quantità di ferro ${ }^{4}$. Tuttavia, è l'inventario dei beni del monastero femminile di S. Giulia di Brescia, a

\footnotetext{
3 R. FARINELLI e R. FRANCOVICH, Potere e attività mineraria nella Toscana altomedievale, in R. Francovich e G. Noyè (eds.), La storia dell'Alto Medioevo italiano alla luce dell'archeologia, Firenze, 1994, p. 443-65; V. LA SALVIA, L'artigianato metallurgico dei Longobardi alla luce delle fonti archeologiche con particolare riferimento alla lavorazione del ferro. Suggerimenti e problemi, in Archeologia medievale, 25, 1998, pp. 22-23.

${ }^{4}$ L'area in questione è nota, infatti, fin dal periodo tardo antico per la persistenza delle attività metallurgiche: la vita di s. Vigilio da Funto ricorda che nel IV secolo lo stesso santo si trovò a dover distruggere una statua del dio Tillino, non casualmente fabbricata in ferro battuto. All'interno della necropoli longobarda di Leno, la tomba 224 si presenta con un ricco corredo che riflette, quindi, lo status elevato dell'inumato. La presenza di un fabbro all'interno di questa necropoli, inoltre, non sembra casuale dal momento che, su circa un migliaio di oggetti, 625 risultano essere spade, sax, coltelli, punte di freccia e di lance, parti di scudi, speroni, armille ed altri piccoli oggetti suntuari in ferro o, comunque, in metallo, sottolineando allo stesso tempo la grande quantità di materia prima disponibile e l'importanza della produzione siderurgica nella medesima zona in epoca longobarda. D'altro canto, presso Bienno, la produzione del ferro, risulta attestata grazie allo scavo di alcune fornaci e scorie di ferro proprio per l'epoca tardo antica e longobarda. In proposito vedi, G. ROSA, Sulle miniere di ferro della Lombardia, in Commentari dell'Ateneo di Brescia, 1843, p. 161-70; V. LA SALVIA, op. cit. (n. 2), p. 68; C. CUCINI TIZZONI, Un forno da ferro longobardo nelle Alpi italiane: Ponte di Val Gabbia - Val Camonica (Bienno, Brescia), in Notiziario di archeologia medievale, 65, 1995, p. 8-9; C. CUCINI TIZZONI e M. TIZZONI, La miniera perduta, Bienno, 1999, p. 93-139 e 141-67; G. ARCHETTI, Ferro e miniere nelle valli bresciane. Il "Centro di documentazione per la storia e l'arte del ferro" e la storiografia recente, in P.P. Poggio, C. Simoni (a cura di), Musei del ferro in Europa e in Italia. La ricerca storica e le esperienze di conservazione e valorizzazione, Atti del convegno (Brescia - Tavernole sul Mella, 24-25 settembre 2004), Brescia, 2006, pp. 43-56.
} 
fornire maggiori informazioni in relazione alla questione in esame. Benché in questo documento non sia descritta direttamente alcuna attività artigianale nel monastero, lo stesso riporta che molti poderi dipendenti, ubicati nelle ricche colline metallifere delle prealpi lombarde, devono canoni in ferro o attrezzi già lavorati, in quantità rilevante: 30 libbre di ferro rendono 8 manentes insediati su una sors della corte di Cassivico; 6o libbre di ferro riscuote da 83 servi la corte di Bradellas in Val Camonica; 20 libbre è il fictum corrisposto, ogni anno, da un manens della corte di Borgonato; 20 vomeri, 3 scuri, una mannaia, 2 forche di ferro (menzionate per la prima volta nella documentazione medievale dell'Italia del Nord) ed altre 100 libbre di ferro vengono dai dipendenti della corte di Griliano; 5 vomeri da tre manentes di Mairano; 4 vomeri e 4 falci sono consegnati alla corte di Odolo; 130 libbre di ferro provengono dal beneficium dell'amministratore Pietro, legato alla corte di Vuassaningus, forse Siniga nel comune di Pisogne. Si tratta, complessivamente, di 340 libbre di ferro, 29 vomeri, 3 scuri, 1 mannaia, 4 falci, 2 forche, un quantitativo che testimonia, così, un'attività artigianale diffusa che si esplica, principalmente, presso le zone ricche di giacimenti minerari ma che, in ogni caso, resta strettamente controllata e legata alle esigenze della struttura economica del monastero. In particolare, la presenza di una discreta quantità di metallo, oltre alla strumentazione in ferro (essenzialmente legata al 'governo' del paesaggio agrario in genere, bosco e incolto compreso, come lascia intendere la presenza di scuri), permette di supporre che all'interno del monastero stesso o almeno delle sue pertinenze, esistessero delle officine in grado di trasformare la materia prima in prodotti finiti, come il caso del grande complesso monastico molisano di S. Vincenzo al Volturno, presso il quale sono state rinvenuti diversi opifici di trasformazione, pare confermare. Inoltre, tanto gli scavi condotti nell'area del monastero quanto l'analisi della formazione storica del suo patrimonio sembrerebbe far propendere per un più precoce inizio della produzione minerario metallurgica nell'area e del relativo interesse delle aristocrazie locali e del monastero stesso in detta attività: già dalla fondazione del 760 , infatti, il re Desiderio, fra i beni mobili ed immobili di spettanza al monastero, ricorda oltre agli strumenti in ferro (ferramenta) anche una corte pubblica presso Roncadelle (de Brada), nei pressi del fiume Mella, poco a sud della città. Per quanto concerne le altre corti menzionate dal polittico, un diploma di Lotario I del 837 è assai importante in quanto riporta possedimenti a Griliano ed in Valcamonica non indicando una nuova accessione della proprietà ma, piuttosto, facendo seguito ad una sua riorganizzazione che parrebbe essere avvenuta proprio negli anni 813-814 attraverso una inchiesta sul campo e non attraverso una semplice revisione documentale ${ }^{5}$.

Una situazione simile si riscontra anche per il monastero di Bobbio. Dal breve memoriationis dell'abate Wala, probabilmente redatto fra 1'833 e 1'835, si comprendono meglio i principi ispiratori dell'organizzazione economica curtense dei monasteri dal momento che lo stesso documento fornisce lo schema della pianificazione delle risorse dei diversi possessi del monastero e le precise indicazioni per la loro gestione: una serie di officine provvedeva alla fabbricazione di tutti i manufatti necessari al normale funzionamento dell'azienda ed ogni settore produttivo era affidato ad un funzionario che, al tempo stesso, era responsabile del lavoro dei servi e dell'approvvigionamento delle materie prime. Infatti, per quel che concerne la produzione siderurgica, Wala suggerisce che «camararius abbatis provideat omnes fabros scutarios [...] et ipse provideat omnia ferramenta». Naturalmente, le singole aziende curtensi, disseminate sul territorio, rispondevano alla casa madre in base alla loro capacità produttiva ed alle dimensioni/particolarità delle risorse locali, così, infatti, Wala: «Gardam deputavit ad oleam, Luliaticam ad ferrum». Da questa corte di Luliatica, nel Pavese, negli anni successivi, provengono differenti manufatti in ferro: dei sette fictales registrati nel breve bobbiese dell'anno 862, uno deve corrispondere come canone annuo cinque vomeri, e lo stesso numero di vomeri è tenuto a prestare ancora al momento della stesura di un secondo breve, nell'883. Non è possibile sapere, invece, donde provenisse il ferro che i dipendenti della corte di Sorlasco, nel Pavese, erano tenuti a trasportare fino a Piacenza. Anche le corti di Luliatica e Sorlasco, tuttavia, sembrano essere state parte del patrimonio primitivo del cenobio. Infatti, un diploma di Berengario del 903, confermando all'abate Teodelassio i privilegi apostolici e i praecepta ottenuti dai re longobardi e dai suoi predecessori (Carlo Magno, Lotario I e II, Carlomanno, Carlo III e Arnolfo), sottolinea quanto antica fosse la proprietà del monastero all'interno di questi distretti produttivi, dal momento che entrambe le corti menzionate nei brevi di IX secolo, sono ricordate anche nel successivo documento di conferma di Berengario. I possedimenti di questi due grandi monasteri non rappresentano, tuttavia, casi eccezionali. La medesima circostanza si ripropone per il monastero milanese di S. Ambrogio che, nel corso del X secolo impone ai suoi servi presso la corte di Limonta, già in suo possesso dall'835 grazie ad una donazione di Lotario I, censi in ferro e per l'abbazia di Nonantola che riceve la sottomissione del fabbro Godeperto nell'anno $907^{6}$.

\footnotetext{
${ }_{5}$ Per quanto detto cfr. V. FUMAGALLI, Strutture materiali e funzioni dell'azienda curtense. Italia del Nord: sec. VIII e IX, in Archeologia medievale, 7, 1980, p. 26; G. PASQUALI, Santa Giulia di Brescia, in A Castagnetti, M. Luzzatti, G. Pasquali e A. Vasina (ed.), Inventari altomedievali di terre, coloni e redditi, Roma, 1979, p. 49-50, 54, 63-65, 69, 71-73; C. Brühl (ed.), Codice diplomatico longobardo, 3, 1, Roma, 1973, n. 33 (Pavia 4 ottobre 760), p. 206-207; T. Schieffer (ed.), Lothari I et Lothari II diplomata, in Monumenta Germaniae Historica, Diplomata Karolinorum, 3, Berolini 1966, n. 35, p. 112-15; G. PASQUALI, Gestione economica e controllo sociale di S. Salvatore-S. Giula dall'epoca longobarda all'età comunale, in C. Stella e G. Brentegani (eds.), S. Giulia di Brescia. Archeologia, arte e storia di un monastero regio dai Longobardi al Barbarossa, Brescia, 1992, p. 133-37; G. ARCHETTI, "Secundum monasticam disciplinam". San Salvatore di Brescia e le trasformazioni istituzionali di un monastero regio, in G. Archetti (a cura di), Desiderio. Il progetto politico dell'ultimo re longobardo, Atti del Primo convegno internazionale di studio (Brescia, 21-24 marzo 2013), Spoleto, 2015 (Centro studi longobardi. Convegni, 1), p. 635-636; inoltre, si consideri che officine sono state rinvenute anche presso il grande complesso monastico di S. Vincenzo al Volturno vedi J. MORELAND, A monastic workshop and glass production at San Vincenzo al Volturno. Molise, Italy, in R. Hodges, J. Mitchell (eds.), San Vincenzo al Volturno. The archaeology, art and territory of an early medieval Monastery, Oxford, 1985, p. 37-6o; R. HODGES, S. LEPPARD, J. MITCHELL, San Vincenzo maggiore and its workshops, in Archeological Monographs of the British School at Rome, 2011, p. 195-253; J. MITCHELL, R. HODGES, S. LEPPARD, A. SEBASTIANI, Le officine di San Vincenzo al Volturno: fasi di produzione e dinamiche di un monastero di IX secolo, in A.C. Quintavalle (a cura di), Medioevo: le officine, Atti del convegno di studi internazionali di Parma, Milano, 2011, p. 114-115; F. MARAZZI, K.D. FRANCIS, L'eredità dell'antico. Tecnologia e produzione in un monastero imperiale carolingio: San Vincenzo al Volturno, in Atti del XI Convegno Internazionale su l'Africa romana, Sassari,1996, p. 1029-1045.

${ }^{6}$ Per i documenti citati cfr. G. TIRABOSCHI, Storia dell'augusta Badia di Nonantola, aggiuntovi il codice diplomatico della medesima, t. 2, Modena, 1785, p. 9 o doc. LXVII (a. 907); Codice diplomatico del monastero di S. Colombano di Bobbio, 1, C. Cipolla (ed.), Roma, 1918, rispettivamente doc. n. XXXVI, 137 ff. e n. LXIII, 184 ff., doc. n. LXXXI, 272; V. FUMAGALLI, op. cit. (n. 5), p. 26; F. CARLI, Il mercato nelletà del Comune, in Storia del commercio italiano, 2 ,
} 
Questo ultimo documento è particolarmente importante in quanto lascia intravedere l'intero andamento del processo economico e, allo stesso tempo, leggere le - mutate - relazioni fra artigianato specializzato e committenza, secondo una prospettiva che vede le strutture monastiche giocare un ruolo centrale nel 'regolare' e definire gli ambiti delle attività produttive. Sulla base del documento, infatti, è possibile supporre che la preoccupazione/necessità di procurarsi strumenti di lavoro abbia, da un lato, spinto l'abate di Nonantola Pietro, a commutare il canone in natura corrisposto da Gudepertus faber, titolare di un podere nel Comasco, con il prodotto della sua attività artigianale, obbligandolo a consegnare ogni anno, entro il mese di aprile, quindici falci prataricias e, dall'altro, il fabbro a cedere porzioni sempre maggiori del suo 'libero' lavoro in cambio della titolarità del possesso terriero. Per quanto concerne le falci, in modo del tutto eccezionale, nel documento citato, ne vengono stabilite anche le dimensioni, permettendone almeno una parziale ed ipotetica ricostruzione. Il documento riporta che le «falces prataricias bonas quindecim cum [...] ferreas eatum [...] sicut necesse est segandum. Sed tale debeant esse [...] ut sint unaquaque longa pedes legitimos doos manualis ad mediocrem hominem, quod sunt duos pedes, semisses quattuor»; ovvero, traducendo in italiano, "ogni falce sia lunga due piedi, e il manico adatto ad un uomo di media statura, cioè lungo due piedi e quattro semissi". Il tipo di falce fienaia, così come presentato nel documento, risulta quindi dotato di un manico con scarso sviluppo in confronto alla lama, secondo uno schema funzionale possibilmente di tradizione "italica", stando a quanto rammenta Plinio Seniore, nella sua Naturalis historia ${ }^{7}$, e diversamente da un altro tipo, con manico più lungo, di possibile origine centro-europea, nell'alveo della tradizione celtica.

La proprietà principale di questo attrezzo è, comunque, quella di consentire il taglio dell'erba non rasoterra ma ad una certa altezza dal suolo e, quindi, di poter essere utilizzato con una mano sola, anche restando in posizione eretta. Tale caratteristica potrebbe essere stata all'origine della sua versatilità funzionale che, sempre secondo quanto indica Plinio, la rendeva utile anche inter vepres, fra gli sterpi, facendone, quindi, uno strumento principe anche per la lotta all'incolto e per il governo della selva, attività quotidiane del lavoro nelle campagne alto medievali. Questa tipologia di falce, tuttavia, ricorda da vicino anche quanto archeologicamente noto grazie ai rinvenimenti di Osterburken e di Runde Berg bei Urach nell'attuale Germania, e mette in luce la presenza, per l'intero alto medioevo, sia di forme specifiche per questa classe di attrezzi, sia di tipi di transizione fra quanto ereditato dal mondo greco-romano e ciò che, invece, pare essere stato tipico della tradizione centro-europea ${ }^{8}$.

A partire dalla fine del secolo VIII secolo, dunque, si assiste ad una tendenza all'accentramento del controllo sulle materie prime che si definisce nei suoi aspetti formali e strutturali, specie in epoca carolingia, e che riguarda non solo i monasteri ma anche contesti produttivi 'laici', come nel caso del 'castello' di Miranduolo, trasformatosi, nel corso dell'alto medioevo, da villaggio aperto a vocazione mineraria in curtis agricola. Un processo archeologicamente ben evidente e rilevabile attraverso il mutamento radicale dell'urbanistica di molti centri demici rurali che 'acquisiscono', a partire dal secolo IX, zone privilegiate all'interno dell'insediamento (sommitali e/o 'semplicemente' circondati da palizzate) in cui si concentrano tanto le attività manifatturiere quanto i prodotti finali (agricolo-alimentari e artigianali). I dati di molta della documentazione scritta dell'Italia centrale e settentrionale segnalano, inoltre, che il rapporto fra artigianato e committenza si istituzionalizza fino a prefigurare la concreta dipendenza del lavoro artigianale, secondo un vero e proprio 'canone' che si ripete, ancora una volta, nell'intera Europa continentale carolingia: il caso dell'abbazia di Lorsch, che nel 788 ricevette come censo 30 chilogrammi di ferro dalla corte del villaggio Weilnau è, infatti, paradigmatica anche della sopraggiunta collateralità di certe attività produttive specializzate rispetto alla produzione agricola, ormai divenuta ovunque primaria9.

I maestri metallurgisti, liberi e ancora in grado di agire come protagonisti in diverse transazioni economiche in pieno VIII secolo, sullo scorcio dello stesso cominciarono,

Padova, 1934, p. 69-70 e G. ROSA, Metallurgia storica bresciana, in Commentari dell'Ateneo di Brescia, 1877, p. 92; Lotharii I et Lotharii II diplomata, op. cit. (n. 5), n. 23, p. 93 .

${ }_{7}$ PLINIO IL VECCHIO, Naturalis historia, F. Semi (ed.), Pisa, 1977, lib. 17, cap. 57, par. 261, 5; si veda anche K.D. WHITE, Agricultural implements of the Roman World, Cambridge, 1967, p. 98-103.

${ }^{8}$ Sullo sviluppo dell'attrezzatura agricola alto medievale vedi, M. BARUZZI, I reperti in ferro dello scavo di Villa Clelia. Note sull'attrezzatura agricola nell'Alto Medioevo, in R. Francovich (ed.), Archeologia e storia del medioevo italiano, Roma, 1987, p. 151-170; J. HENNING, Frühgeschichtliche Landwirtschaft Südosteuropas: Vom Großgrundbesitz zur Großgrundwirtschaft, in F. Horst, B. Krüger (eds.), Produktivkräfte und Produktionsverhältnisse in ur- und frühgeschichtlicher Zeit, Berlino, 1985, p. 301-08; J. HENNING, Zur Datierung von Werkzeug- und Agrargerätenfunden im germanischen Landnahmegebiet zwischen Rhein und oberer Donau (Der Hortfund von Osterburken), in Jahrbuch des römisch-germanischen Zentralmuseums Mainz, 32, 1985, p. 570-94; J. HENNING, Südosteuropa Zwischen Antike und Mittelalter, Berlin, 1987; F. ZAGARI, V. LA SALVIA, Aspetti della produzione metallurgica longobarda, in Paolo Diacono e il Friuli alto medievale (secc. VI-X), Atti del XIV Congresso internazionale di studi sull'alto Medioevo (Cividale del Friuli-Bottenicco di Moimacco, 24-29 settembre 1999), Spoleto, 2001, p. 871-85; V. LA SALVIA, F. ZAGARI, Cultura materiale e tradizione tecnica: la metallurgia del ferro dei Longobardi in Italia, in I Longobardi dei Ducati di Spoleto e Benevento, Atti del XVI Congresso internazionale di studi sull'alto Medioevo (Spoleto $20-23$ ottobre 2002 - Benevento 24-27 ottobre), Spoleto, 2004, p. 968-989.

${ }_{9}^{9}$ In proposito si veda, M. CIMA, Archeologia del ferro. Sistemi, materiali e processi dalle origini alla Rivoluzione Industriale, Brescia, 1991, p. 40, 121; U. ZIMMERMANN, Mittelalterlicher Bergbau auf Eisen, Blei und Silber - Begrenzte Mittel und zahlreiche Veränderungen, in G. Magnusson (ed.), The Importance of Ironmaking The Importance of Ironmaking: Technical Innovation and Social Changes, Papers presented at the Norgberg Conference on May 8-13, 1995, Stockholm, 1995, p. 275. Su Miranduolo (ed altri casi toscani) e lo sviluppo dell'economia carolingia, M. VALENTI, Insediamento e strutture di potere in Italia centrale: il caso toscano, in M. Valenti e Ch. Wickham (eds.), Italy, 888-962: a turning point. Italia, 888-962: una svolta, IV Seminario Internazionale (Cassero di Poggio Imperiale a Poggibonsi, 4-6 dicembre 2009), Turnhout, 2013, p. 271-276, 288, 299; ID., Conclusioni, Ibidem, p. 427-446; ID., Miranduolo in Alta Val di Merse (Chiusdino - SI). Archeologia su un sito di potere del medioevo toscano, Firenze, 2008; ID., L'insediamento altomedievale delle campagne toscane. Paesaggi, popolamento e villaggi tra VI e X secolo, Firenze, 2004; V. LA SALVIA, Gli indicatori della produzione metallurgica presso il sito di Miranduolo (Chiusdino, Si) con particolare riferimento alle fasi altomedievali, in VI Congresso di archeologia medievale (L'Aquila, 12-15 settembre 2012), Firenze, 2012, p. 640-43; V. LA SALVIA, L. ANGUILANO, La metallurgia del ferro fra VII e VIII secolo a Miranduolo: nota preliminare, in VII Congresso di Archeologia Medievale (Lecce, 9-12 settembre 2015), Firenze, 2015, p. 313-317. 
invece, a cedere porzioni sempre maggiori dei propri diritti ai rappresentanti del potere e dell'aristocrazia (anche religiosa), cui devolvevano il frutto del loro lavoro specializzato sotto forma di censi prestabiliti (in materia prima e/o in oggetti e ferramenta) in cambio, quasi sempre, del godimento di rendite fondiarie. Ciò se da un lato lascia intendere, che non vi fu nel corso dell'alto Medioevo alcuna generalizzata penuria di metallo (come messo in luce dallo studio, datato ma puntualissimo, di Liborio, Marinig, e Storti del 1989) ${ }^{10}$, dall'altro, indica che la produzione si era ormai fortemente orientata/focalizzata per rispondere agli interessi preponderanti della grande proprietà fondiaria cui la riorganizzazione economica dei centri monastici non fu certamente estranea e, al contrario, appare aver giocato un ruolo fondamentale nella definizione dei nuovi modi di controllare l'accesso alla materie prime e al territorio in genere.

${ }^{10}$ Si fa riferimento a C. LIBORIO, T. MARINIG, A. STORTI, Regiones augusteae X et XI: ritrovamenti di manufatti in ferro databili dall'età augustea all'età altomedievale, in Dal basso fuoco all'altoforno. Atti del 1 Simposio Valle Camonica 1988: La siderurgia nell'antichità, in Sibrium, 20, 1989, p. 23-37, specie p. 26. 